\title{
Experimental studies to evaluate the cellular and molecular mechanisms of a traditional polyherbal anti-asthmatic preparation UNIM-352 in rats: a reverse pharmacology approach
}

\author{
Nishant Rai, Kavita Gulati, Arunabha Ray \\ Pharmacology, Vallabhbhai Patel Chest Institute, University of Delhi, Delhi, India
}

Background: Bronchial asthma is a chronic inflammatory disease of the airways characterized by airway inflammation, airflow obstruction, airway hyperresponsivness and airway remodeling. UNIM-352 is a polyherbal formulation used in Indian traditional medicine for the treatment of bronchial asthma. The present study evaluated the effects of UNIM-352 in experimental model of airway inflammation and remodeling, with an aim to elucidate the cellular and molecular mechanisms to validate its use in asthma.

Methods: The airway inflammation studies, rats were immunized with ovalbumin (OVA) and alum on day 1 and then challenged on day 14 with OVA. For airway remodeling studies, rats were immunized with OVA and alum on day 1, and then challenged with antigen aerosols from day 15 to 21. In both models, after $24 \mathrm{~h}$ of OVA challenge blood and bronchoalveolar lavage (BAL) fluid were collected and the effects of UNIM-352 were assessed on various markers of (a) airway inflammation and immunity, (b) airway remodeling, (c) bronchial hyperresponsiveness to methacholine using whole body plethysmography, (d) oxidative stress parameters. In both experiments, the treatment groups were: control (vehicle), UNIM-352 (200 and $400 \mathrm{mg} / \mathrm{kg}$ ), and prednisolone $(10 \mathrm{mg} / \mathrm{kg})$.

Results: The results showed that UNIM-352 attenuated the levels of TNF-alpha, IL-4, IgE, GM-CSF, IL-13 and TGFbeta, whereas histone deacetylase levels were elevated, as compared to the control group, in both blood and BAL fluid. Further, cytology of blood and BAL fluid revealed that UNIM-352 reduced the eosinophil and neutrophil counts, the effector cells in asthma. UNIM-352 also reduced the hydroxyproline content, an efficient marker of collagen production in lung tissue. In addition, UNIM-352 treated rats showed reduced degree of inflammation, goblet cell hyperplasia and subepithelial fibrosis, the important markers of remodeling on histopathological examination of lung tissues. UNIM-352 also attenuated 8-OHdG and 8-isoprostane levels in both body fluids, which suggested protective effect of UNIM-352 against reactive oxygen species and oxidative DNA damage. Further, the polyherbal agent reduced the bronchial hyperreactivity and airflow obstruction in response to methacholine exposure.

Conclusions: The results suggest anti-inflammatory, immunomodulatory, anti-remodeling, anti-oxidant and antispasmogenic mechanisms of UNIM-352 and this could contribute to its observed beneficial effects in bronchial asthma. 